\title{
Diagnostic delay in migraine with aura
}

\author{
G. Viticchi • M. Bartolini $\cdot$ L. Falsetti • \\ J. Dolcini · S. Luzzi • L. Provinciali • \\ M. Silvestrini
}

(C) Springer-Verlag Italia 2013

\begin{abstract}
Migraine with aura (MWA) is a common headache, characterized by short-lasting neurological signs preceding an headache attack with migraine characteristics. We have recently performed several investigations about time-delay for migraine without aura diagnosis (MWoA). Aim of this study was to compare the time necessary to obtain a correct diagnosis in MWA and MWoA patients. We enrolled 31 consecutive patients affected by MWA and 62 age- and sex-matched patients affected by MWoA. All subjects were submitted to a face-to-face interview about the time-delay from symptoms' onset and a correct migraine typology diagnosis, the number of specialists consulted and the instrumental examinations performed. Independent samples and repeated measures $t$ test, MannWhitney $U$ were performed to compare the variables of the cases with the matched controls. No significant differences were found among the collected variables. Diagnostic delay was significantly different $(p<0.05)$, resulting lower in patients affected by MWA than in those with MWoA. In fact, subjects affected by MWA had a mean diagnostic delay of 6.70 years $(\mathrm{SE} \pm 1.5$ ), while patients affected by MWoA had a mean interval of 10.7 years $(\mathrm{SE} \pm 1.20$ ). Patients affected by MWA present a significant lower delay for the formulation of a correct diagnosis with respect to subjects with MWoA. This could be probably due to the
\end{abstract}

G. Viticchi $(\bowtie) \cdot$ M. Bartolini · J. Dolcini · S. Luzzi •

L. Provinciali - M. Silvestrini

Neurological Clinic, Department of Experimental and Clinical

Medicine, Marche Polytechnic University, Via Conca 71,

60020 Ancona, Italy

e-mail: giovanna.viticchi@libero.it

L. Falsetti

Internal and Subintensive Medicine, Ospedali Riuniti Ancona,

Ancona, Italy fact that MWA patients develop major alarm reactions related to visual symptoms and look for an earlier appointment with a specialist medical center.

Keywords Migraine with aura - Diagnostic delay · Headache management

\section{Introduction}

Migraine with aura (MWA) is a subtype of headache characterized by various short-lasting neurological signs preceding an headache attack with migraine characteristics. The most common symptoms are visual signs, such as flashes of light, blind spots or tingling. Less frequently, it is possible to find motor symptoms as arm or leg paralysis or sensitive signs, such as diffuse parasthesias. Frequently, MWA can be misleading for specialists other than neurologists, and could be confounded with other pathologies, as epileptic crisis or multiple sclerosis [1]. This fact could cause an alarm in the patient who is very often submitted to several useless instrumental examinations before a correct diagnosis. In the past, we have investigated the time-delay for a correct diagnosis in subjects affected by migraine without aura (MWoA) showing that these patients are frequently waiting several years before arriving to a definite diagnosis [2]. Moreover, we demonstrated that this type of delay is closely related with the number and typology of consulted specialists and performed instrumental examinations [3]. Aim of this study was to evaluate the time requested to obtain a correct diagnosis in patients affected by MWA, to compare it with the time needed in subjects affected by MWoA, and to verify if there is any relevant difference between these two typologies of headache. 
Table 1 Baseline characteristics of the two groups of patients

\begin{tabular}{llll}
\hline & MWA group $(n=31)$ & MWoA group $(n=62)$ & Student's or $\chi^{2}$ test \\
\hline Age $( \pm$ SD), years & $37.71( \pm 14.12)$ & $37.71( \pm 14.00)$ & n.s. \\
Sex $($ males, $\%)$ & 48.4 & 48.4 & n.s. \\
Number of specialists $( \pm$ SE) & $0.45( \pm 0.11)$ & $0.69( \pm 0.11)$ & n.s. \\
Number of exams $( \pm$ SE) & $0.97( \pm 0.23)$ & $1.24( \pm 0.16)$ & n.s. \\
Diagnostic delay $( \pm$ SE) & $6.70( \pm 1.5)$ & $10.7( \pm 1.2)$ & $p<0.05$ \\
\hline
\end{tabular}

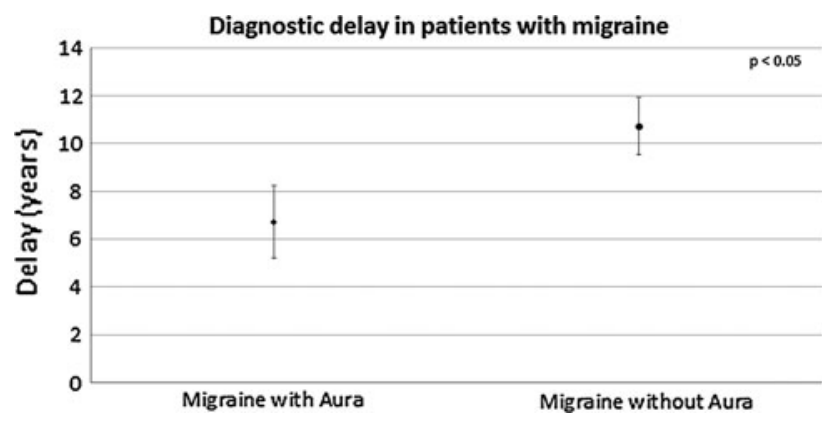

Fig. 1 Mean difference of diagnostic delay between MWA and MWoA

\section{Methods}

During a 1-year period, we enrolled 31 consecutive patients arrived to our Headache Center affected by MWA and 62 age- and sex-matched subjects affected by MWoA. In each patient, we put a first diagnosis of migraine. For this study, we decided to select only subjects with visual aura to obtain a homogeneous sample. We submitted all the sampled people to an in-depth face-to-face interview regarding the time delay from symptoms' onset, age, sex, education, number of performed instrumental/laboratory exams and consulted specialists before the correct diagnosis. Continuous variables were compared with independent samples and repeated measures $t$ test and Mann-Whitney $U$ test. Dichotomous variables were analyzed with Chi-square test. Analysis was performed with SPSS 13.0 for Windows systems.

\section{Results}

The two groups resulted homogeneous as shown in Table 1. Diagnostic delay resulted significantly lower in the MWA group than in the MWoA one [6.70 years $(\mathrm{SE} \pm 1.5)$ vs. 10.7 years $(\mathrm{SE} \pm 1.20), p<0.05$ ] (Fig. 1).

\section{Discussion}

Our findings show that the patients affected by MWA have a significantly lower delay in diagnosis formulation with respect to patients with $\mathrm{MWoA}$. In our previous studies, we investigated the possible reasons for diagnostic delay in MWoA, and we speculated that the most important point was probably patients' late referral to a headache center. The most relevant problems related to this malpractice are the loss of resources due to useless examinations' performance and, in some cases, the incorrect therapeutic approaches.

MWA is a benign disease characterized by surprising and alarming symptoms for most of subjects. In several cases, patients refer to an emergency department in the suspect of a serious illness, such as stroke or a multiple sclerosis attacks. In other cases, when asking their general practitioner, they are quickly referred to a specialistic neurological evaluation. All these elements can contribute to a more rapid diagnostic pathway for MWA with respect to MWoA and, consequently, to a better disease management.

The delay in migraine diagnosis remains one of the most relevant problems for headache specialists. Often these patients arrive to our centers with an history of chronic headache, bringing several misconceptions about their state of health and, more generally, with a lack of trust in medical therapy. In our experience, these elements are less frequently observed in MWA, probably for the better casemanagement.

Conflict of interest I certify that there is no actual or potential conflict of interest in relation to this article.

\section{References}

1. Applebee A (2012) The clinical overlap of multiple sclerosis and headache. Headache 52(Suppl 2):111-116

2. Viticchi G, Silvestrini M, Falsetti L et al (2011) Time delay from onset to diagnosis of migraine. Headache 51(2):232-236

3. Viticchi G, Silvestrini M, Falsetti L et al (2011) The role of instrumental examinations in delayed migraine diagnosis. Neurol Sci 32(Suppl 1):S143-S144 